\section{(6) OPEN ACCESS}

\title{
The intervertebral disc contains intrinsic circadian clocks that are regulated by age and cytokines and linked to degeneration
}

\author{
Michal Dudek, 1,2 Nan Yang, 1,2 Jayalath PD Ruckshanthi, 1,2 Jack Williams, 1,2 \\ Elzbieta Borysiewicz, ${ }^{1}$ Ping Wang, ${ }^{1}$ Antony Adamson, ${ }^{1}$ Jian Li, ${ }^{1}$ John F Bateman, ${ }^{3}$ \\ Michael R White, ${ }^{1}$ Raymond P Boot-Handford, ${ }^{2}$ Judith A Hoyland, ${ }^{4,5}$ \\ Qing-Jun Meng ${ }^{1,2}$
}

\begin{abstract}
Handling editor Tore K Kvien
- Additional material is

published online only. To view please visit the journal online (http://dx.doi.org/10.1136/ annrheumdis-2016-209428)
\end{abstract}

For numbered affiliations see end of article.

Correspondence to Dr Qing-Jun Meng, Faculty of Life Sciences, University of Manchester, A.V. Hill Building, Oxford Road, Manchester M13 9PT, UK;

qing-jun.meng@manchester. ac.uk and Professor Judith A Hoyland, Centre for Tissue Injury and Repair, Faculty of Medical and Human Sciences, University of Manchester, Stopford Building, Oxford Road, Manchester, M13 9PT, UK ; judith.a.hoyland@ manchester.ac.uk

JAH and Q-JM are cocorresponding authors.

Received 23 February 2016 Revised 6 June 2016 Accepted 9 July 2016 Published Online First 2 August 2016

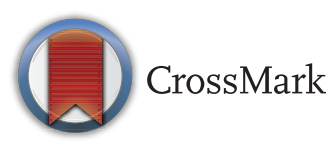

To cite: Dudek M, Yang N, Ruckshanthi JPD, et al. Ann Rheum Dis

2017:76:576-584

\section{ABSTRACT}

Objectives The circadian clocks are internal timing mechanisms that drive $\sim 24$-hour rhythms in a tissuespecific manner. Many aspects of the physiology of the intervertebral disc (IVD) show clear diurnal rhythms. However, it is unknown whether IVD tissue contains functional circadian clocks and if so, how their dysregulation is implicated in IVD degeneration. Methods Clock gene dynamics in ex vivo IVD explants (from PER2:: luciferase (LUC) reporter mice) and human disc cells (transduced with lentivirus containing Per2::luc reporters) were monitored in real time by bioluminescence photon counting and imaging. Temporal gene expression changes were studied by RNAseq and quantitative reverse transcription (qRT)-PCR. IVD pathology was evaluated by histology in a mouse model with tissue-specific deletion of the core clock gene Bmal1.

Results Here we show the existence of the circadian rhythm in mouse IVD tissue and human disc cells. This rhythm is dampened with ageing in mice and can be abolished by treatment with interleukin- $1 \beta$ but not tumour necrosis factor $\alpha$. Time-series RNAseq revealed 607 genes with 24-hour patterns of expression representing several essential pathways in IVD physiology. Mice with conditional knockout of Bmal1 in their disc cells demonstrated age-related degeneration of IVDs.

Conclusions We have established autonomous circadian clocks in mouse and human IVD cells which respond to age and cytokines, and control key pathways involved in the homeostasis of IVDs. Genetic disruption to the mouse IVD molecular clock predisposes to IVD degeneration. These results support the concept that disruptions to circadian rhythms may be a risk factor for degenerative IVD disease and low back pain.

\section{INTRODUCTION}

The circadian clocks are internal timing mechanisms which drive 24 -hour rhythms in physiology and behaviour. In mammals, the central pacemaker suprachiasmatic nuclei (SCN) in the hypothalamus synchronises peripheral clocks in most major body organs. ${ }^{1-3}$ Circadian rhythms coordinate tissuespecific physiology with light/darkness, rest/activity, feeding cycles and body temperature fluctuations. ${ }^{14}$ Disruptions to circadian rhythms (during ageing or in shift workers) have been linked to increased risk of diseases (eg, obesity, diabetes, cardiovascular disease and osteoarthritis). ${ }^{5}{ }^{6}$ At the molecular level, the circadian clock consists of a network of transcriptional activators (Clock, Bmal1) and repressors (Per1/2 and Cry1/2) organised in a negative feedback loop. ${ }^{6}$ This core oscillator generates 24-hour rhythms in the expression of its core components and a myriad of clock-controlled genes. Depending on the tissue, expression of $3 \%-16 \%$ of the whole transcriptome exhibits a circadian rhythm. $^{7}$

The spine is comprised of bony vertebral bodies alternating with fibrocartilagenous intervertebral discs (IVD). IVD degeneration is among the most prevalent musculoskeletal disorders affecting one in five people under 60 and more than half of the people above 60 years of age. ${ }^{8}$ Low back pain, which is often associated with IVD degeneration, is the number one cause of years lived with disability in the developed countries. ${ }^{9}$ Existing evidence suggests that the IVD is a highly rhythmic tissue, experiencing a diurnal cycle of higher loading (activity phase), ${ }^{10}{ }^{11}$ followed by a period of low-load recovery (resting phase). Under high load, the pressurised interstitial fluid flows to regions of lower pressure through the outer annulus fibrosus (AF) and the cartilaginous end plate (CEP), resulting in decreased disc height, AF outward bulging and an increase in osmolarity of the central gelatinous nucleus pulposus (NP). During the recovery period, the process is reversed by high osmotic pressure inside the disc causing fluid flow to the NP. ${ }^{12}$ Exchange of nutrients/metabolites that occurs with fluid flow during this cycle maintains disc cell homeostasis. $^{13}$

Consistent with the rhythmic nature of IVD tissue, shift work (a factor known to disrupt circadian rhythms) was reported to be associated with higher risk of low back pain (LBP) and IVD degeneration. ${ }^{14-18}$ We have previously shown that environmental disruption of circadian rhythm in mice, when combined with high fat diet, leads to degeneration of the lumbar IVD tissue in mice. ${ }^{19}$ More recently, changes in the expression of circadian clock genes have been identified in rat IVD tissues following passive smoking (a risk factor for LBP). ${ }^{20}$ However, no studies have examined whether IVD cells express intrinsic circadian clocks, how these 
IVD clocks are regulated, what their targets are and whether genetic disruption to the IVD clock impact on tissue homeostasis and susceptibility to degeneration.

In this study, we systemically characterised the molecular circadian clock mechanisms in mouse and human IVD tissue/cells. Moreover, by generating a tissue-specific Bmal1 KO mouse model, our study provides the first genetic evidence linking a core clock factor to IVD degeneration.

\section{RESULTS}

\section{Intervertebral disc possesses a functional, temperature entrainable circadian clock}

To test whether the IVD contains a molecular circadian clock capable of driving circadian rhythm of gene expression, we monitored the dynamics of PER2::Luc protein in IVD explant cultures isolated from PER2::Luc reporter mice. ${ }^{21}$ Real-time bioluminescence photon counting demonstrated robust circadian rhythm of PER2::Luc activity which lasted for more than 5 days, with a period of $23.93 \pm 0.10$ hours (mean $\pm S E M, n=6$, figure $1 \mathrm{~A}$ ). As the IVD comprises two distinct cell types, the NP and AF cells, we wanted to know if both regions exhibit circadian rhythms. Live imaging of the mouse IVD explants using high-sensitivity electron multiplying (EM)-CCD camera revealed rhythmic PER2::Luc signals from both AF and NP cells (see online supplementary videos S1-3). To extend these studies to humans, primary human NP cells were transiently transfected with a vector carrying the luciferase gene under the control of the Per2 promoter. This approach revealed cell-autonomous circadian oscillations of Per2::luc expression, indicating the operation of a functional clock machinery in these human disc cells (figure 1B). Immunohistochemistry (IHC) staining of human NP tissue sections using antibodies against BMAL1 and CLOCK confirmed the presence of these essential circadian clock components in human discs (figure 1C).

One of the key properties of a peripheral circadian clock is their ability to respond to time cues that are controlled by the SCN clock, such as hormones or changes in body temperature. Since the IVDs are not vascularised or innervated (except in pathological conditions), ${ }^{22}$ we hypothesised that daily body temperature oscillations may be a mechanism of clock entrainment for IVDs. To test this, IVD explants from the same mouse were placed in different incubators programmed to have oppositely phased cyclic temperature changes for 4 days $\left(38.5^{\circ} \mathrm{C}\right.$ for 12 hours $/ 35.5^{\circ} \mathrm{C}$ for 12 hours, or vice versa), before returning to a constant $37^{\circ} \mathrm{C}$. As a control, another IVD explant from the same mouse was incubated under constant $37^{\circ} \mathrm{C}$. The PER2:: Luc rhythms in IVD explants were all in similar circadian phase for the first 3 days before the temperature protocol (figure 1D). Once the antiphasic protocol was introduced, the oscillations were driven $180^{\circ}$ out of phase with each other. Interestingly, the antiphasic oscillations were maintained for at least three more days after the tissues were released to constant temperature. In
A

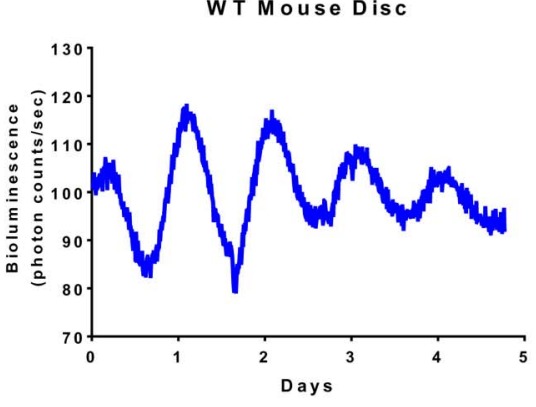

C
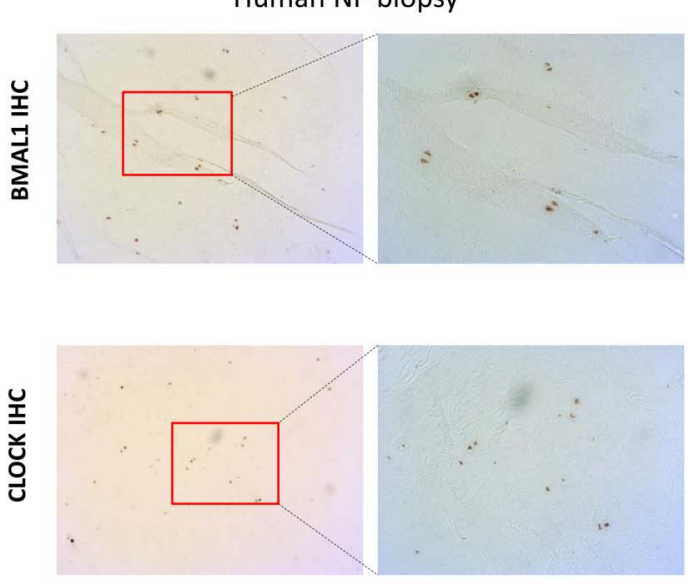
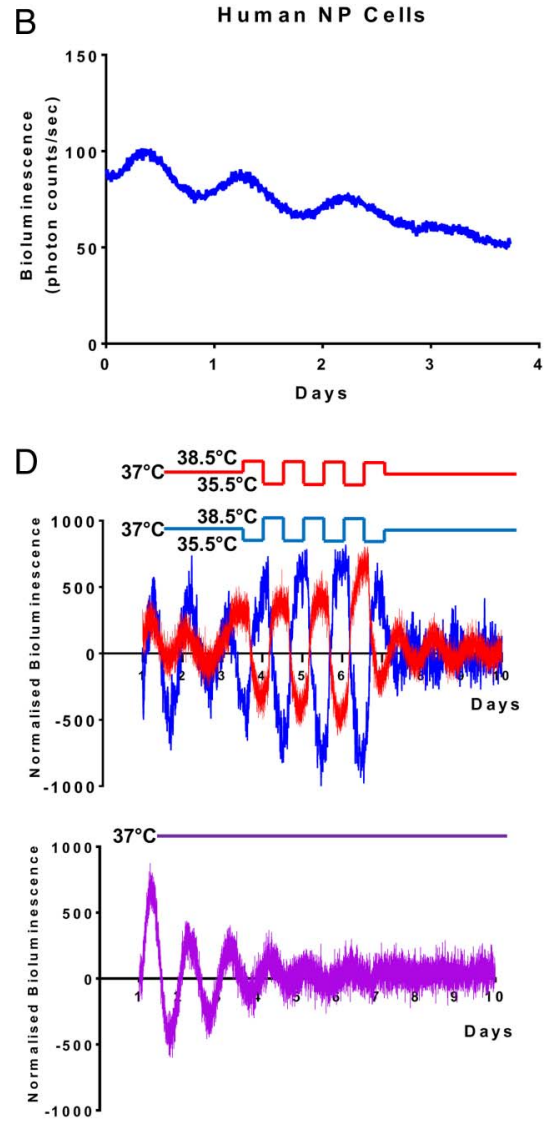

Figure 1 Intervertebral discs (IVDs) possess an autonomous circadian clock. (A) Representative PER2::Luc bioluminescence trace of mouse IVD explant culture (period=23.93 \pm 0.247 hours; mean $\pm S D ; n=6$ ). (B) Representative trace of human nucleus pulposus (NP) cells transduced with a Per2:: luc reporter (period=22.52 \pm 0.39 hours; mean $\pm S D ; n=3$ ). (C) IHC of BMAL1 and CLOCK on NP biopsy of human IVDs (magnification $5 \times$ left, 10x right); $n=3$. (D) Temperature entrainment ( $n=4)$. Two IVD explant cultures (represented by red and blue traces) from the same animal were held under antiphase temperature cycles (alternating 12-hour cycles of $38.5^{\circ} \mathrm{C} / 35.5^{\circ} \mathrm{C}$; baseline temperature $=37^{\circ} \mathrm{C}$ ). Third IVD explant culture from the same animal was kept at a constant temperature of $37^{\circ} \mathrm{C}$ (purple trace below). 

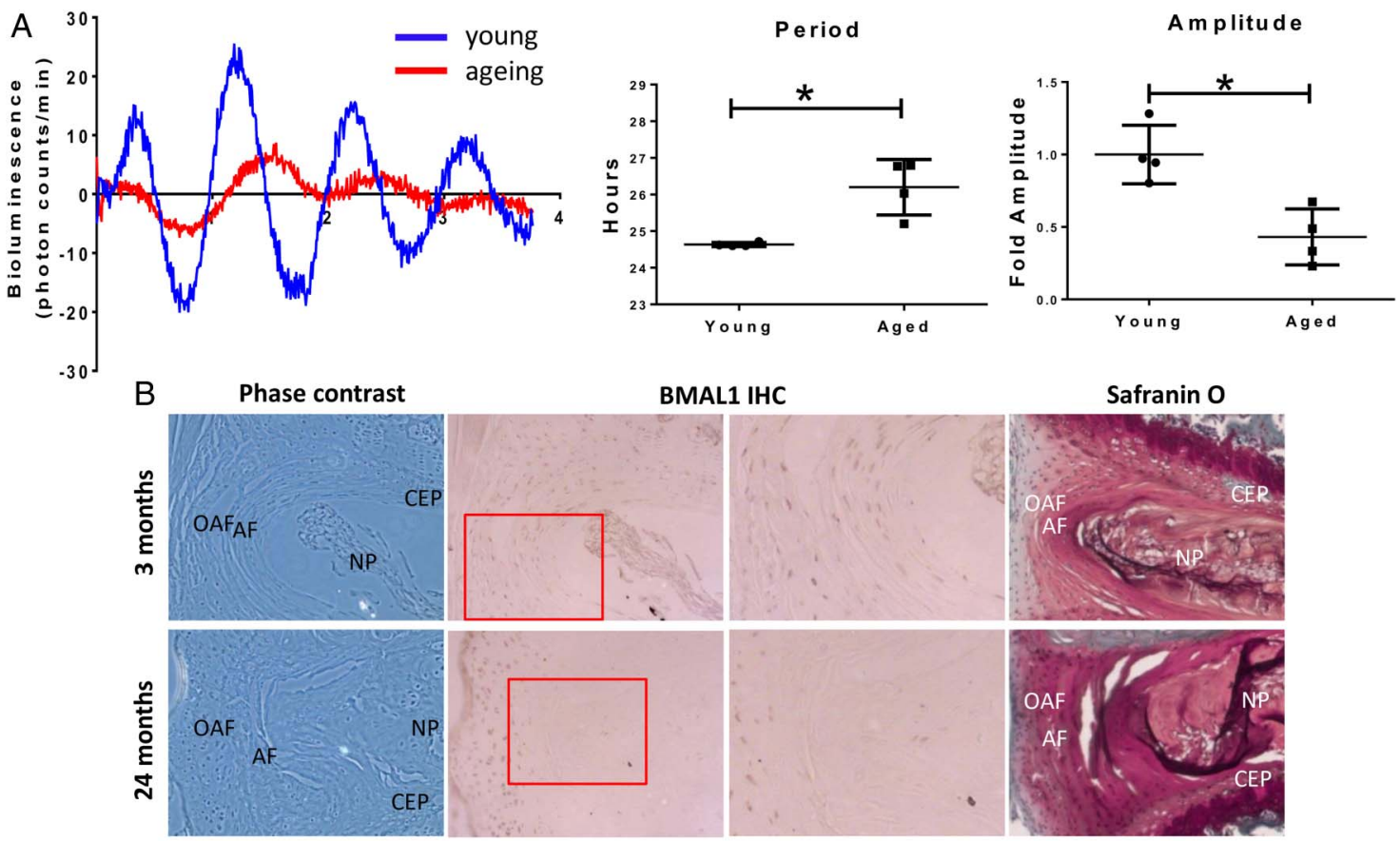

BMAL1 IHC

Safranin 0
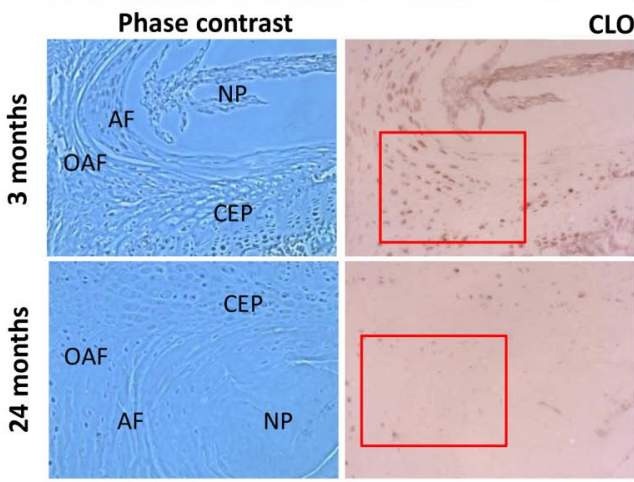

CLOCK IHC
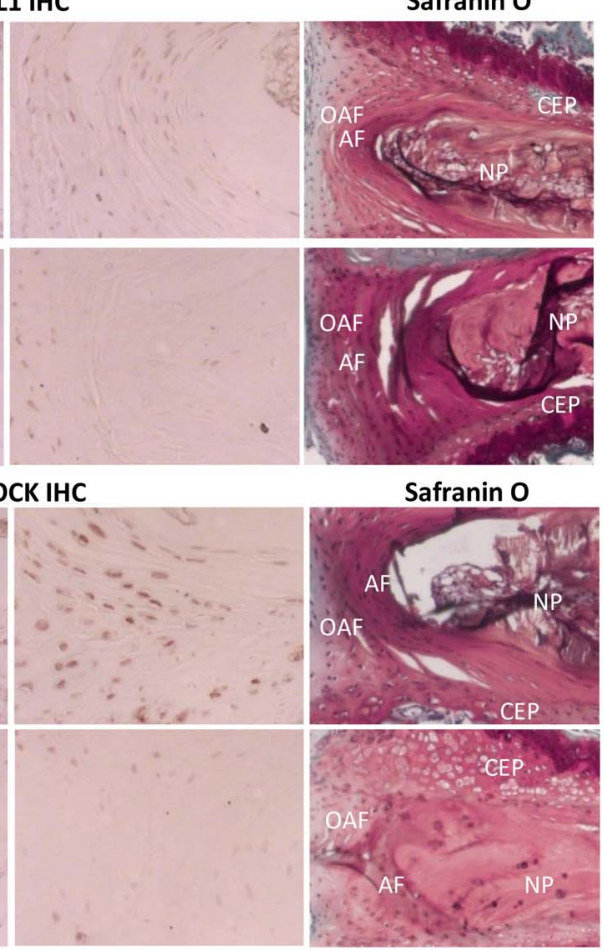

Figure 2 Circadian rhythm of IVD is dampened during ageing. (A) Representative bioluminescence traces of young (2 months) and ageing (12 months) IVDs from PER2::Luc mice. The period was significantly lengthened in older mice $(p<0.05)$ and the amplitude was significantly dampened $(p<0.05)$ (two-tailed non-parametric Mann-Whitney test; $n=4)$; $(B)$ IHC of BMAL1 and CLOCK on young (3 months) and aged $(24$ months) mouse IVDs; $n=4$. Magnification 10x. The Safranin 0 staining panel on the right was included to ease visualisation of the different structures of the IVD. AF, annulus fibrosus; CEP, cartilaginous end plate; IVD, intervertebral disc; NP, nucleus pulposus; OAF, outer annulus fibrosus.

contrast, the IVD explant that remained at constant temperature gradually lost its ability to oscillate by day 7 , mainly due to desynchronisation in culture (figure 1D). These results clearly indicate that temperature cycles that approximate body temperature changes are capable of entraining the circadian phase of the IVD oscillation and enhancing the oscillation amplitude.

\section{Ageing affects the circadian rhythm of IVDs}

Daily systemic time cues in body temperature and hormone release are known to be altered with ageing. ${ }^{23}$ In addition, intrinsic properties of the clock oscillator could deteriorate with age as well. ${ }^{23} 24$ Indeed, we have previously demonstrated that the amplitude of circadian oscillations in cartilage and tendon tissues dampen with ageing. ${ }^{25} 26$ Therefore, we hypothesised that circadian rhythms may change in ageing disc, compromising the daily control of IVD physiology. To assess this, we compared the oscillations of PER2::Luc expression in mouse IVD explant cultures from animals aged 2 and 12 months (see figure $2 \mathrm{~A}$ and online supplementary Video S1). The amplitude of oscillations in IVDs from 12 months old mice was severely reduced (by $\sim 60 \%$ ) as compared with 2-month-old mice. Additionally, the average period of oscillations was significantly lengthened by
1.6 hours in IVDs from 12-month-old mice (figure 2A). IHC staining showed decreased expression of the core circadian transcription factors BMAL1 and CLOCK in 12-month (see online supplementary figure S1) and 24-month-old mice as compared with 2-month-old mice (figure 2B). These data demonstrate that the IVD clock becomes dysregulated with ageing.

\section{The circadian rhythm of IVD is disrupted by interleukin-1及 in a NF- $\kappa$ B-dependent manner}

Chronic inflammation is a known factor associated with IVD degeneration and lower back pain. ${ }^{27}$ To investigate the effects of catabolic cytokines on disc circadian clock, we treated IVD explants from the PER2::Luc reporter mice with interleukin (IL) $1 \beta$, lipopolysaccharide (LPS) and tumour necrosis factor (TNF) $\alpha$. Tissues were under continuous bioluminescence recording. Treatment with IL-1 $\beta$ (or LPS, see online supplementary figure S2A) resulted in complete disruption of the PER2::Luc circadian rhythm, associated with significant changes of clock genes (Bmal1, Per2 and Nr1d1) (see figure 3A and online supplementary figure S3). The disrupted rhythm could be reinstated by dexamethasone (an anti-inflammatory glucocorticoid, figure 3A) or IL-1RA (an antagonist of IL-1, see online supplementary figure S2B), but not by 

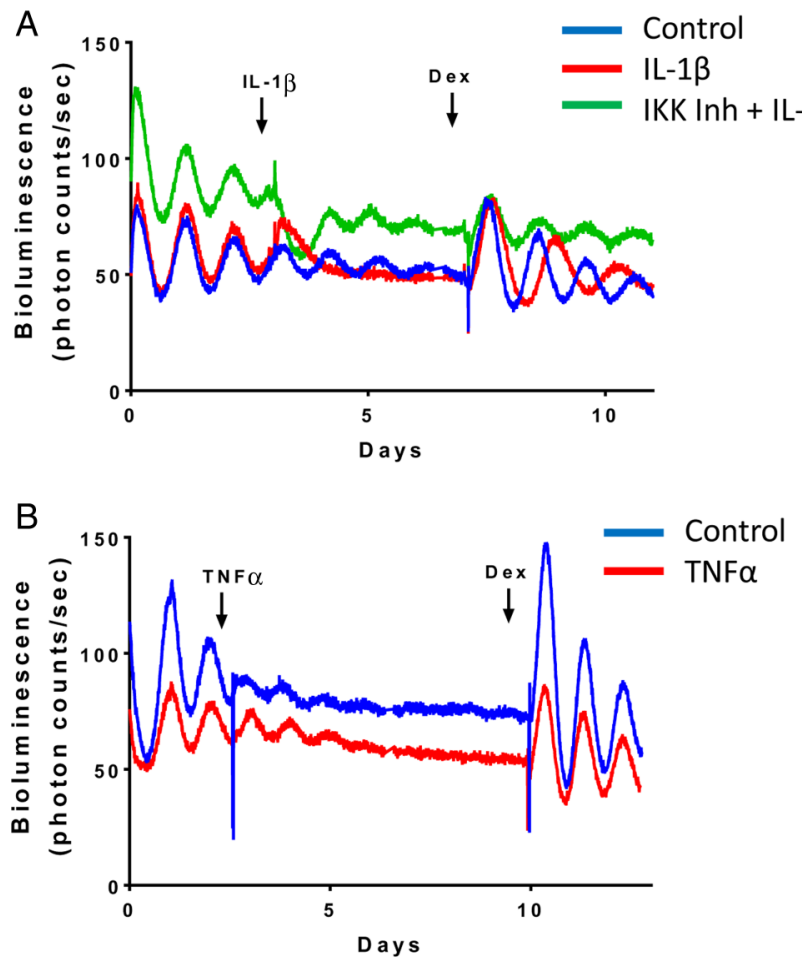
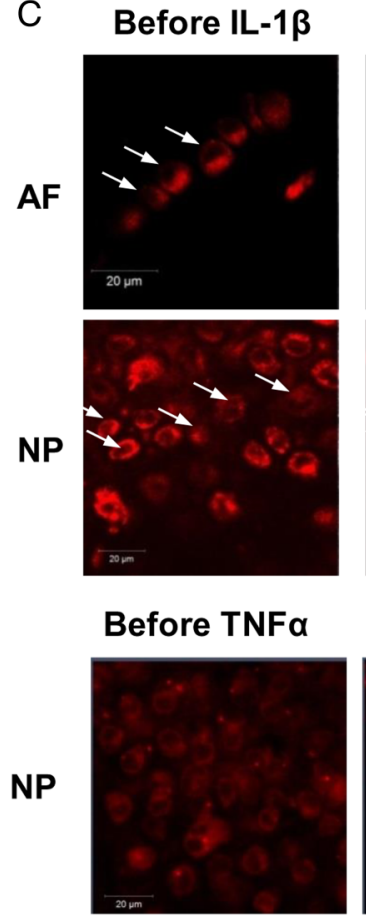

After IL-1 $\beta$
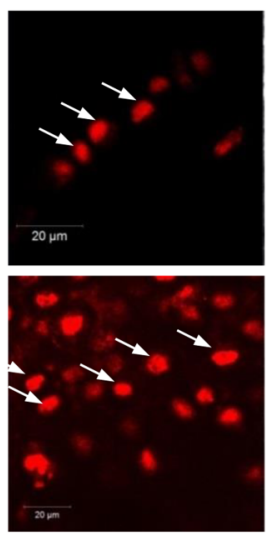

After TNFa

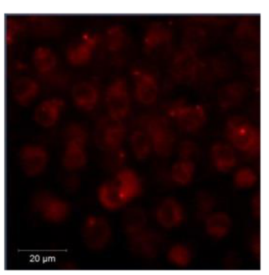

D

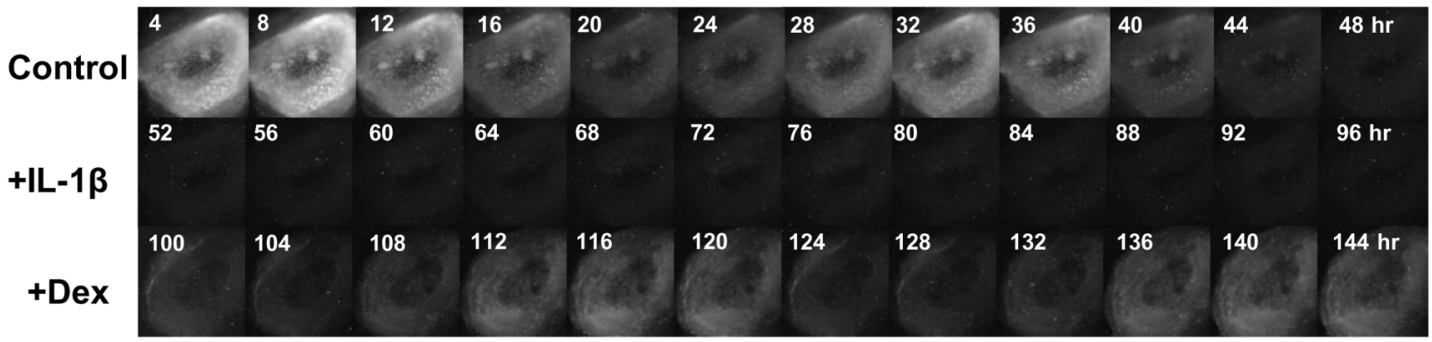

Figure 3 IL1 $\beta$, but not TNF $\alpha$, disrupts the circadian rhythm of IVDs. (A) Representative bioluminescence traces of PER2::Luc mouse IVD explants. Arrows indicate time of treatment with IL-1 $\beta(5 \mathrm{ng} / \mathrm{mL})$, inhibitor of kappaB kinase (IKK) inhibitor (BMS-345541, $10 \mu \mathrm{M})$ and dexamethasone $(100 \mathrm{nM})$. Red trace-treated with IL-1 $\beta$, green trace - pretreated with IKK inhibitor before addition of IL-1 $\beta$, blue trace-vehicle control; $n=3$. (B) Representative bioluminescence traces treated with TNF $\alpha$ (red trace, $40 \mathrm{ng} / \mathrm{mL}$ ) or control (blue trace). Arrows indicate time of treatments; $\mathrm{n}=3$. (C) Live fluorescence imaging of p65DsRed reporter in mouse IVDs by confocal microscopy before and after treatment with IL-1 $\beta$ or TNF $\alpha$. Scale bar $20 \mu \mathrm{m}$. Arrows indicate the nuclei. (D) Live bioluminescence imaging of an IVD tissue from PER2::Luc mouse, treated with IL-1 $\beta$ (at 48 hours), followed by dexamethasone (at 96 hours). AF, annulus fibrosus; IL, interleukin; IVD, intervertebral disc; NP, nucleus pulposus; TNF, tumour necrosis factor.

forskolin (a clock synchronising agent without anti-inflammatory properties, see online supplementary figure S2C). Nuclear factor kappa $\mathrm{B}(\mathrm{NF}-\mathrm{\kappa} \mathrm{B})$ is one of the classical pathways through which IL-1 $\beta$ can mediate its effects. To evaluate the involvement of NF- $\mathrm{BB}$, we used the IKK1/2 inhibitor BMS-345541 to block the activation of NF- $\mathrm{B}$. The clock-disrupting effect of IL-1 $\beta$ was blocked by pretreating the IVD explant with BMS-345541, sup-

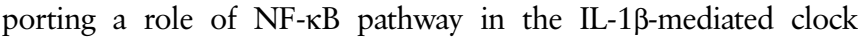
disruption. In contrast to IL-1 $\beta$, treatment of IVD explants with TNF $\alpha$ had no effect on their circadian rhythms (figure 3B). In contrast, both IL-1 $\beta$ and TNF $\alpha$ elicited a strong induction of NF- $\kappa B$ signalling in a lung epithelial cell line, suggesting a possible cell-type-specific response (see online supplementary figure S2D). Next, we took advantage of a transgenic mouse strain expressing the $\mathrm{p} 65$-DsRedXP protein fusion construct ${ }^{28}$ to observe the nuclear translocation of p65, one of the major components of the NF- $\kappa \mathrm{B}$ complex. Live imaging showed that treatment of IVD explants with IL-1 $\beta$ caused rapid nuclear translocation of p65 both in AF and NP cells. However, addition of TNF $\alpha$ (up to $40 \mathrm{ng} / \mathrm{mL}$ ) had no effect on p65 translocation (figure $3 \mathrm{C}$ ).
There are at least two potential mechanisms through which IL-1 $\beta$ could disrupt the IVD circadian rhythm. Individual cells may still have robust clocks but become desynchronised, with their clocks being in different phases, leading to reduced oscillation amplitude, or individual cells may have lost their pacemaking properties. To distinguish between these two possibilities, we used a high-sensitivity EM-CCD camera to visualise the PER2::Luc bioluminescence signals from individual cells in the presence or absence of IL-1 $\beta$. Consistent with the lack of effect of forskolin, this imaging approach revealed loss of bioluminescence at single cell level, excluding the desynchronisation hypothesis (see figure 3D and online supplementary video S2). Therefore, disruption to the IVD clock could be a hitherto undiscovered response to proinflammatory cytokines.

\section{Identification of the first IVD circadian transcriptome}

Circadian clocks in different tissues exert their local functions through regulating diverse yet highly tissue-specific set of target genes. To reveal the extent of rhythmic genes in IVD tissue under physiological conditions, we performed a time-series 


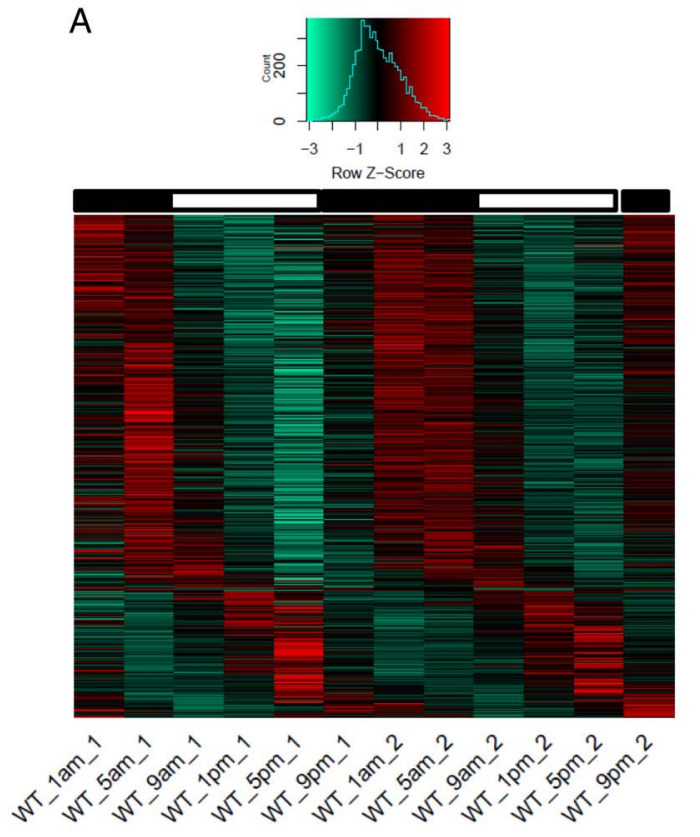

C

RNAseq
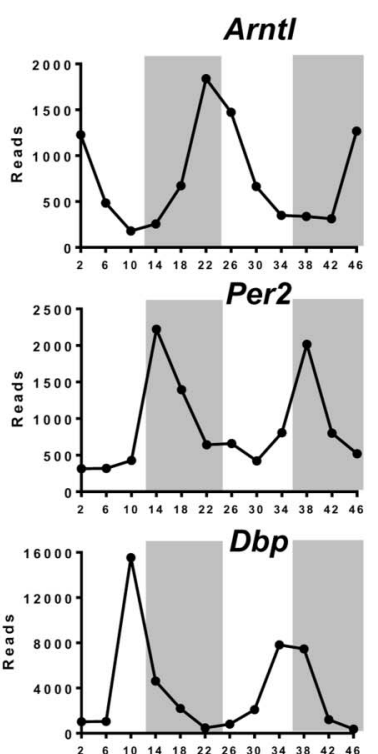

Follistatin

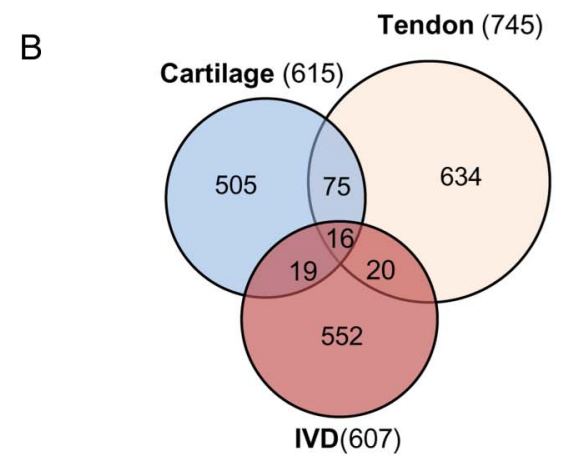

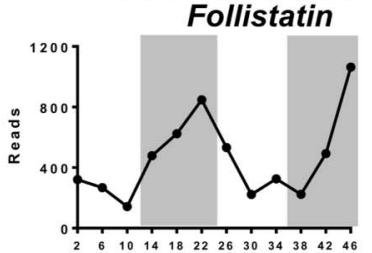

Timp4

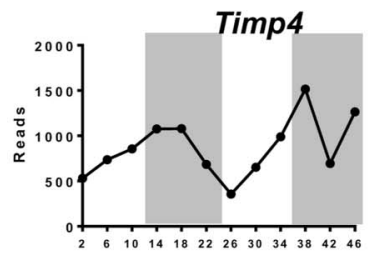

qPCR

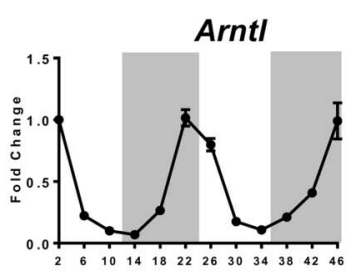

Per2

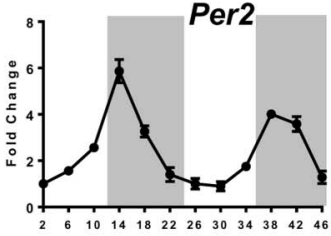

Dbp

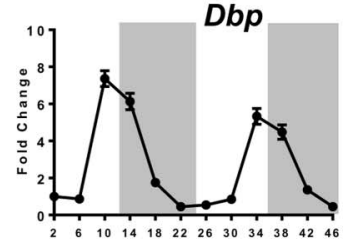

Follistatin

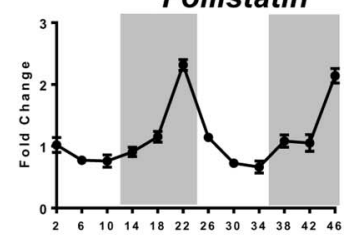

Timp4

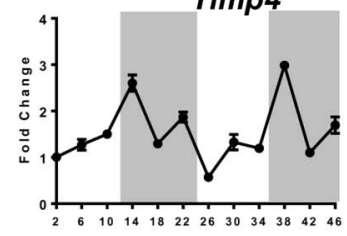

Figure 4 Circadian transcriptome in mouse intervertebral disc (IVD) identified by time-series RNA sequencing. (A) Heat map depicting the expression patterns of the 607 rhythmic genes (3.5\% of the IVD transcriptome) identified by JTKCycle. Genes were organised according to timing of peak expression. White bars represent the day; black bars represent the night. (B) Venn diagram comparing the number of rhythmic genes of IVD, cartilage and tendon. (C) qPCR validation of time-dependent expression of clock genes (Bmal1, Per2 and Dbp) and target genes (Follistatin and Timp4) in mouse IVDs normalised to Gapdh. Mean and SEM ( $\mathrm{n}=6)$. Grey shadow indicates the night phase.

RNAseq study using IVD tissues (collected every 4 hours for 48 hours) from mice kept in 12-hour light/12-hour darkness. We used a well-recognised JTKCycle ${ }^{29}$ algorithm to pick out rhythmic genes. Using $\mathrm{P}_{\text {adjust }}<0.05$ as a cut-off, we identified 607 genes $(3.5 \%$ of expressed genes in IVD) with rhythmic 24-hour expression patterns (see figure 4A and online supplementary table S1). Further phase clustering analysis of these rhythmic genes using $\mathrm{R}$ package revealed four main clusters (see online supplementary figure S4), with more than $70 \%$ of these genes peaking at night time points (representing the active phase of mouse). Gene ontology (GO)-term analysis using topGO revealed dozens of overrepresented functional groups with an adjusted $\mathrm{p}<0.01$, including 'fatty acid metabolic process', 'circadian rhythm', 'intracellular protein transmembrane transport', 'intrinsic apoptotic signalling pathway', 'carboxylic acid metabolic process' and 'response to endoplasmic reticulum stress'. We next compared the IVD rhythmic gene list with that of the mouse cartilage and tendon we published earlier. $^{25} 26$ There was a very small number of genes (6\%-16\%) overlapping between any two of these skeletal tissues, with only 16 genes common to all three, supporting the tissue-specific function of the peripheral clocks (figure 4B). Of these 16 common genes, 8 were core circadian clock genes. The expression profiles of canonical clock genes (Bmal1, Per2, Dbp) and selected target genes Follistatin (a bone morphogenetic proteins (BMP) antagonist) ${ }^{30}$ and Timp4 (a tissue inhibitor of matrix metalloproteinase (MMPs) $)^{31}$ relevant to IVD physiology and catabolism were validated by temporal quantitative reverse transcription (qRT)-PCR in mouse IVD tissues (see figure 4C and online supplementary figure S5).

\section{Targeted deletion of Bmal1 causes age-dependent IVD degeneration}

Bmal1 is an essential circadian clock component for the generation of 24-hour rhythms. The global Bmal1 knockout mouse shows multitissue pathologies, including ectopic calcification of IVDs. ${ }^{32}$ However, the severe disruption to whole body circadian rhythms confounds interpretation of phenotype. To evaluate the function of local IVD clocks, we produced a conditional KO mouse model (Col2a1-Bmal1 KO, conditional knockout (cKO)) with a cell-type-specific abolition of the transcription factor Bmal1 in $\alpha 1$ (II) collagen-expressing cells, including NP and AF cells, and chondrocytes. ${ }^{33}$ We have previously shown that the central SCN clock and behavioural locomotion rhythms in the 

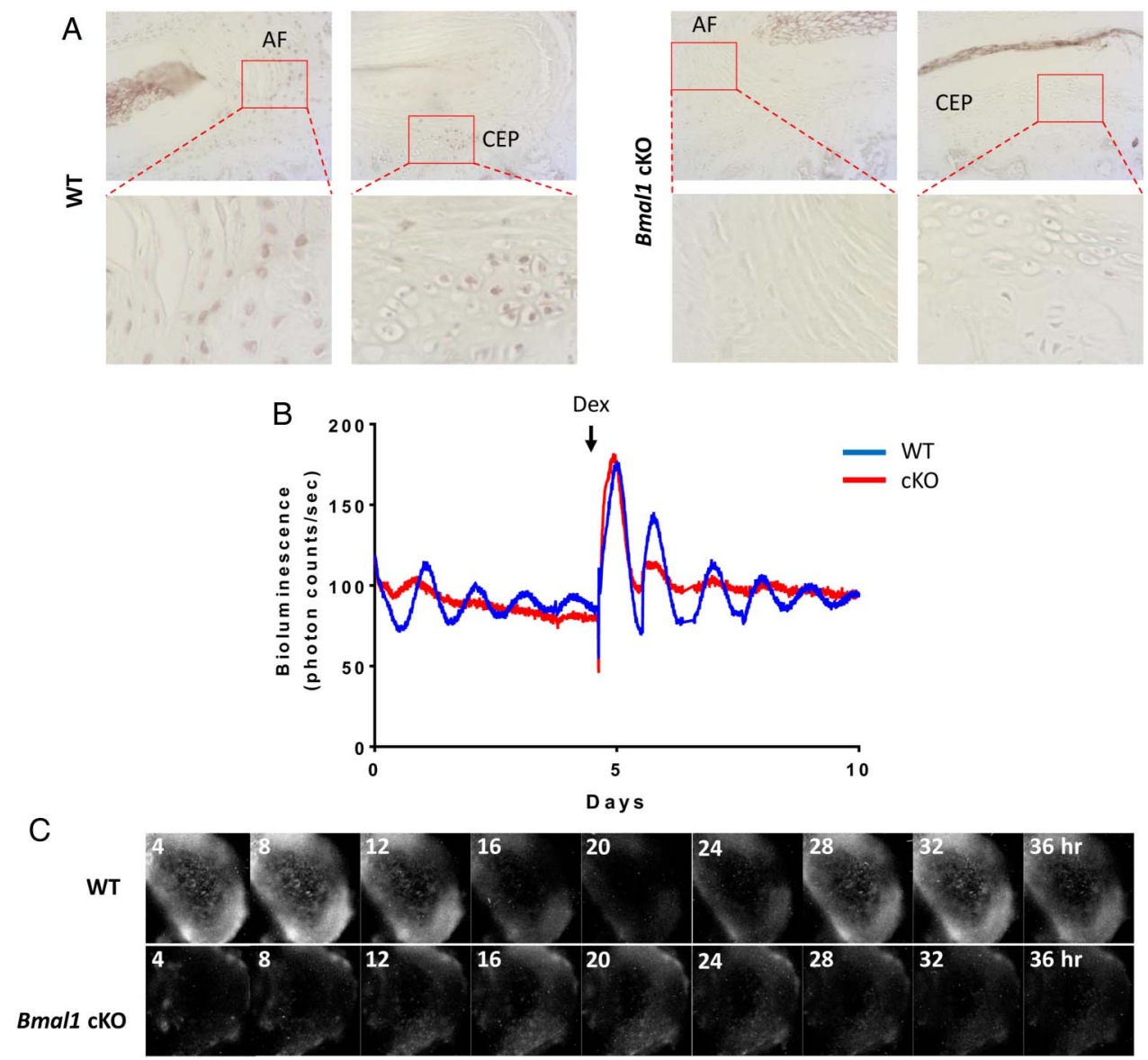

Figure 5 Conditional deletion of Bmal1 in Col2a1-expressing cells results in disruption of the circadian rhythms in mouse intervertebral discs (IVDs). (A) IHC of BMAL1 in 3-month-old wild type (WT) and KO mice (magnification: upper panels 10x and lower panels 40x); $n=3$. (B) Representative bioluminescence traces of WT (blue) and Bmal1 CKO (red) mouse IVD explant cultures; $\mathrm{n}=6$. Arrow indicates treatment with dexamethasone. (C) Live bioluminescence imaging of IVDs from WT and Bmal1 cKO IVDs from mice on a PER2::Luc background.

cKO mice are not affected. ${ }^{33}$ IHC staining of IVDs confirmed loss of BMAL1 expression in the majority of the AF cells and chondrocytes of the CEP in cKO mice (figure 5A). The cKO mouse was crossed with the PER2::Luc mouse to enable real-time tracking of clock rhythms. Photon counting of PER2::Luc bioluminescence demonstrated a lack of circadian oscillations in the cKO IVDs, with no response to dexamethasone treatment (figure $5 \mathrm{~B}$ ). Bioluminescence imaging of the cKO IVDs confirmed lack of circadian oscillations of PER2::Luc in both AF and NP cells (see figure $5 \mathrm{C}$ and online supplementary video $\mathrm{S} 3$ ).

Histological analysis revealed early signs of degeneration of the lumbar IVDs in cKO mouse at 6 months of age, such as thinning of the growth plate of vertebral body (figure 6A), and gradual disappearance of the CEP (see online supplementary figure S6). At 12 months, there was widespread degeneration of lumbar IVDs in cKOs. Bone bridges appeared within the growth plate, the CEP was almost completely replaced by bone (figure $6 \mathrm{~A}$, black arrow) and the height of the disc was significantly reduced in $\mathrm{CKO}$ IVDs (figure 6A). In addition, staining with Safranin $\mathrm{O}$ and picrosirius red revealed disorganisation of the outer annulus structure and signs of fibrosis (with organised collagen bundles) appearing at the periphery of the IVDs (figure $6 \mathrm{~A}-\mathrm{C}$, asterisk). Finally, using X-ray studies, the $\mathrm{cKO}$ mice showed clear signs of calcification and narrowing of spaces between vertebrae at 6 months (in tail IVDs, data not shown) and 12 months (in lumbar IVDs, figure 6C). No signs of degeneration were evident in age-matched wild type (WT) mice up to the age of 12 months (figure 6B). However, similar degenerative changes to the cKO mutants were visible in WT mice at 24 months of age (see online supplementary figure S7), suggesting the possibility that loss of Bmal1 and/or circadian rhythm in IVD cells leads to accelerated ageing of the tissue. Terminal deoxynucleotidyl transferase dUTP nick end labeling (TUNEL) assay and qPCR were performed to explore the underlying mechanisms for the observed phenotype. There were no obvious signs of apoptosis, although significant upregulation of catabolism-related genes (Adamts1, Adamts5, Adamts15 and Follistatin) was observed in cKO IVDs (see online supplementary figures S8 and 9). Together, these results indicate the essential role of the locally expressed core clock factor BMAL1 in IVD homeostasis, loss of which led to profound tissue degeneration.

\section{DISCUSSION}

Low back pain is among the most prevalent spinal diseases associated with increasing age, with over $80 \%$ of the UK population predicted to experience back pain within their lifetime. Progressive degeneration of the IVD tissue, partly caused by increased catabolism driven by inflammatory/catabolic cytokines, is a major contributing factor in $\mathrm{LBP}^{34}$ It has long been known that the physiology of IVD is under strong influence by a diurnal rhythm associated with the rest/activity cycles, that is, daily cycles of loading (activity phase) and low-load recovery (resting phase). ${ }^{10-13}$ Exchange of nutrients/metabolites that occurs with fluid flow during this cycle maintains disc cell 


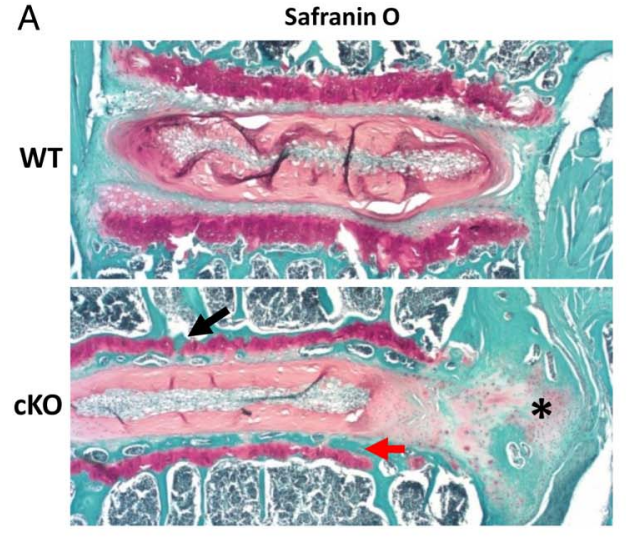

B
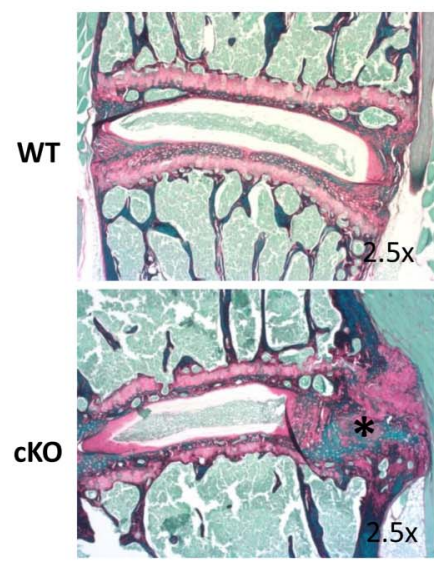

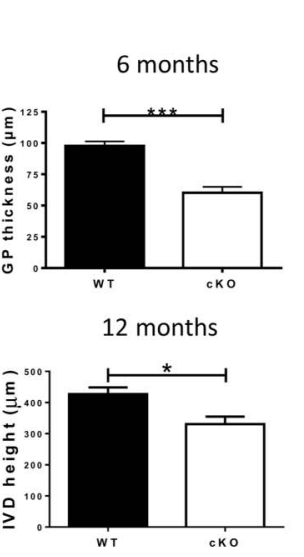

Picrosirius red/brightfield

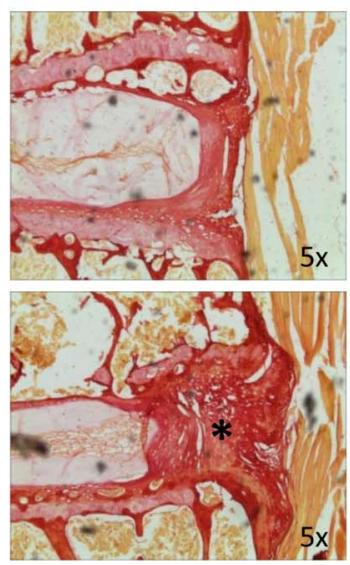

C

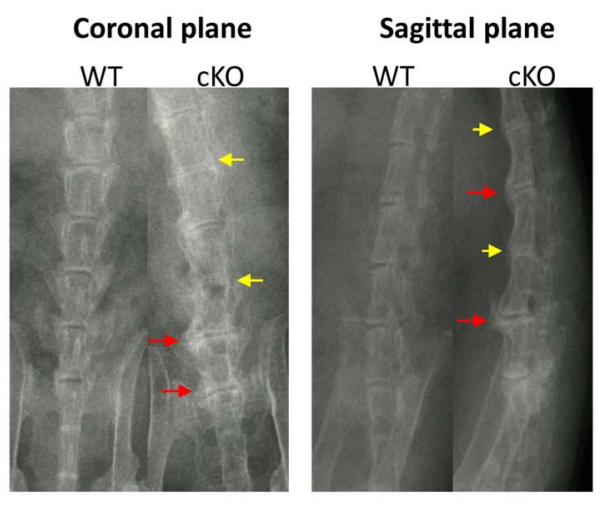

Picrosirius red/polarised light
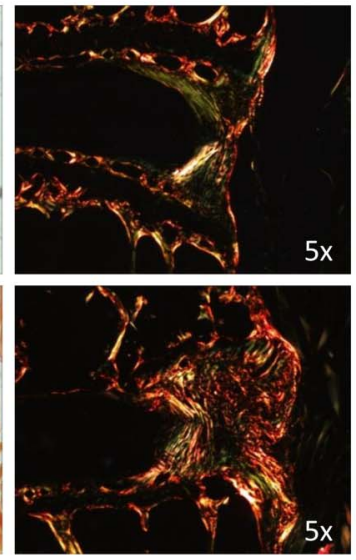

Figure 6 Loss of Bmal1 leads to degeneration of IVDs and cartilaginous tissues of the spine. (A) Safranin 0 staining of 12-month-old WT and Bmal1 cKO mouse lumbar IVDs; $n=4$. Red arrow—loss of cartilaginous end plate (CEP); black arrow—fragmentation of growth plate; *—fibrosis (magnification 2.5x). Analysis of the intervertebral disc (IVD) height and growth plate thickness was shown (two-tailed non-parametric Mann-Whitney test; $n=4){ }^{*} p<0.05 ;{ }^{* *} p<0.001$. (B) Picrosirius red staining of lumbar IVDs from 12-month-old WT and Bmal1 cKO mouse showing organisation of collagen (magnification $2.5 \times$ left and $5 \times$ right panels); $n=4$. Images were visualised under brightfield or polarised light. (C) $X$-ray radiography of 12-month-old WT and Bmal1 cKO mouse spines; $\mathrm{n}=3$. Yellow arrows-calcification of IVDs; red arrows-calcification of tissues surrounding the IVDs.

homeostasis. Recent epidemiological and experimental studies have linked shift work (in humans) and chronic disruption of circadian rhythms (in mice) to higher risk of IVD degeneration. $^{14} 15 \quad{ }^{17-19}$ However, our study represents the first critical analysis of the molecular and cellular mechanisms of the IVD clock under physiological and pathological conditions. Using the clock gene reporter mouse/cell models, as well as a conditional Bmal1 KO mouse model that had disrupted IVD clock, we established autonomous circadian clocks in mouse and human IVD cells that respond to temperature cycles, dampen with age and become dysregulated by catabolic cytokines. Genetic disruption to the mouse IVD molecular clock predisposes to IVD degeneration. Global Bmal1 KO also showed a phenotype in the skeletal system, including the spine. However, our conditional KO model allows us to conclude the essential role of locally expressed BMAL1 or circadian rhythm in maintaining IVD homeostasis. These results support the notion that disruptions to circadian rhythms during ageing or in shift workers may be a contributing factor for the increased susceptibility to degenerative IVD diseases and low back pain.

We also revealed for the first time the circadian transcriptome of the IVD tissue. Of particular interest are the genes and pathways that have been previously implicated in IVD physiology and pathology, such as genes involved in matrix homeostasis/ repair (eg, Follistatin, Timp4, Adamts1, Adamts5, Adamts15 and Adam17), ${ }^{30}{ }^{31}$ mitochondria function and fatty acid metabolism (eg, Pex1, Pex2, Pex5, Pex15, Adipoq, Adipor2 and Fasn). ${ }^{35}$ Although glucose and anaerobic glycolysis represent major metabolic pathways in IVD, there is evidence that mitochondria in the NP are functional and they retain the capacity to metabolise fatty acids through mitochondrial oxidative metabolism. ${ }^{35}$ Other relevant pathways include endoplasmic reticulum (ER) stress and apoptosis (eg, Aifm1, Atf6, Chac1, Bak1, Bbc3, Opa1 and Fas). ${ }^{37}$ The diverse clock-controlled pathways identified by this approach implicate circadian rhythm as a critical regulatory mechanism for IVD biology.

Using IVD tissue explants, we have identified the disruption of the circadian clock in IVD as hitherto undiscovered response to proinflammatory cytokines. Similar clock disruptions by inflammatory cytokines have been found in other cell types, such as in macrophages, ${ }^{39}$ synovial fibroblasts ${ }^{40}$ and chondrocytes. $^{28}$ The involvement of NF- $\mathrm{kB}$ pathway in mediating the effects of IL-1 is consistent with our earlier findings in chondrocytes, where NF- $\kappa \mathrm{B}$ interferes with the core clock complex to disrupt circadian pacemaking. ${ }^{28}$ Given the diverse pathways controlled by the IVD clock, cytokine-mediated circadian disruption may be involved in driving key aspects of the catabolic response of IVD to chronic inflammation. Therefore, there is 
the possibility of stabilising IVD clock rhythm as a novel strategy to combat tissue catabolism. Although the concentration we used for IL-1 $\beta(5 \mathrm{ng} / \mathrm{mL})$ in these tissue explant studies was higher than that in degenerative IVD $(\sim 50 \mathrm{pg} / \mathrm{mL})$, this dose is in line with most ex vivo/in vitro studies. We also identified a lack of response of the IVD clock (and cartilage clock) ${ }^{28}$ to $\mathrm{TNF} \alpha$, possibly due to the defective NF-кB nuclear translocation. These findings suggest that IL- 1 and TNF $\alpha$ may act on distinct downstream pathways and regulate different target genes within the IVD, as seen in chondrocytes. In SW1353 chondrocyte-derived cells, catabolic genes such as IL-6, BMP-2, MMP13 and cyclooxygenase (COX)-2 only respond to IL-1, with almost no response to TNF $\alpha .{ }^{41}{ }^{42}$ Such results are intriguing because we have shown that IL-1 $\beta$ plays a more prominent role in driving disc degeneration than TNF $\alpha^{43}{ }^{44}$ Therefore, antiinflammatory drugs that selectively target IL-1 are more likely to bring therapeutic benefits.

In conclusion, our results provide a firm basis for future studies that aim to elucidate the functional implication and therapeutic potential of the human IVD circadian rhythm in health and disease of the spine.

\section{Author affiliations}

${ }^{1}$ Faculty of Life Sciences, University of Manchester, Manchester, UK

${ }^{2}$ Wellcome Trust Centre for Cell Matrix Research, University of Manchester, Manchester, UK

${ }^{3}$ Murdoch Children's Research Institute, Parkville, Victoria, Australia

${ }^{4}$ Faculty of Medical and Human Sciences, Centre for Tissue Injury and Repair, University of Manchester, Manchester, UK

${ }^{5}$ NIHR Manchester Musculoskeletal Biomedical Research Unit, Manchester Academic Health Science Centre, Manchester, UK

Contributors Q-JM and JAH designed the experiments. MD, NY, JPDR, JW, EB, $A A, J L$ and JFB conducted the experiments and acquired the data. MD, NY, JPDR, $J W, E B, A A, J L, J F B, P W, M R W, R P B H, J A H$ and Q-JM analysed the data. Q-JM and JAH wrote the manuscript.

Funding This work was funded by a Medical Research Council (MRC) UK Career Development Award (G0900414, to Q-JM); an Arthritis Research UK Senior Research Fellowship Award (20875, to Q-JM); an MRC project grant (MR/K019392/1, to Q-JM and RPB-H); a Wellcome Trust (UK) Core funding grant (088785/Z/09/Z) to the University of Manchester Wellcome Trust Centre for Cell Matrix Research. Consumables for processing of human IVD and isolation and culture of human IVD cells were funded by the National Institute for Health Research Manchester Musculoskeletal Biomedical Research Unit.

Competing interests None declared.

Patient consent Obtained.

Ethics approval NRES Committee North West.

Provenance and peer review Not commissioned; externally peer reviewed.

Open Access This is an Open Access article distributed in accordance with the terms of the Creative Commons Attribution (CC BY 4.0) license, which permits others to distribute, remix, adapt and build upon this work, for commercial use, provided the original work is properly cited. See: http://creativecommons.org/licenses/ by/4.0/

\section{REFERENCES}

1 Hastings $M H$, Reddy $A B$, Maywood ES. A clockwork web: circadian timing in brain and periphery, in health and disease. Nat Rev Neurosci 2003;4:649-61.

2 Partch CL, Green CB, Takahashi JS. Molecular architecture of the mammalian circadian clock. Trends Cell Biol 2014;24:90-9.

3 Reppert SM, Weaver DR. Coordination of circadian timing in mammals. Nature 2002;418:935-41.

4 Bass J, Takahashi JS. Circadian integration of metabolism and energetics. Science 2010;330:1349-54.

5 Dudek M, Meng QJ. Running on time: the role of circadian clocks in the musculoskeletal system. Biochem J 2014;463:1-8.

6 Takahashi JS, Hong HK, Ko CH, et al. The genetics of mammalian circadian order and disorder: implications for physiology and disease. Nat Rev Genet 2008:9:764-75.
7 Zhang $\mathrm{R}$, Lahens $\mathrm{NF}$, Ballance $\mathrm{HI}$, et al. A circadian gene expression Atlas in mammals: implications for biology and medicine. Proc Natl Acad Sci USA 2014;111:16219-24.

8 Boden SD, Davis DO, Dina TS, et al. Abnormal magnetic-resonance scans of the lumbar spine in asymptomatic subjects. A prospective investigation. J Bone Joint Surg Am 1990;72:403-8.

9 Global Burden of Disease Study C. Global, regional, and national incidence, prevalence, and years lived with disability for 301 acute and chronic diseases and injuries in 188 countries, 1990-2013: a systematic analysis for the Global Burden of Disease Study 2013. Lancet 2015;386:743-800.

10 Haschtmann D, Stoyanov JV, Ferguson SJ. Influence of diurnal hyperosmotic loading on the metabolism and matrix gene expression of a whole-organ intervertebral disc model. J Orthop Res 2006;24:1957-66.

11 Malko JA, Hutton WC, Fajman WA. An in vivo magnetic resonance imaging study of changes in the volume (and fluid content) of the lumbar intervertebral discs during a simulated diurnal load cycle. Spine 1999;24:1015-22.

12 Matsumoto T, Kawakami M, Kuribayashi K, et al. Cyclic mechanical stretch stress increases the growth rate and collagen synthesis of nucleus pulposus cells in vitro. Spine 1999:24:315-19.

13 van der Veen AJ, van Dieën JH, Nadort A, et al. Intervertebral disc recovery after dynamic or static loading in vitro: is there a role for the endplate? J Biomech 2007:40:2230-5.

14 Elfering A, Semmer N, Birkhofer D, et al. Risk factors for lumbar disc degeneration: a 5-year prospective MRI study in asymptomatic individuals. Spine 2002;27:125-34.

15 Kaila-Kangas L, Kivimäki $M$, Härmä $M$, et al. Sleep disturbances as predictors of hospitalization for back disorders-a 28-year follow-up of industrial employees. Spine 2006;31:51-6.

16 Leino-Arjas P, Kaila-Kangas L, Kauppinen T, et al. Occupational exposures and inpatient hospital care for lumbar intervertebral disc disorders among Finns. Am J Ind Med 2004;46:513-20.

17 Rajaratnam SM, Arendt J. Health in a 24-h society. Lancet 2001;358:999-1005.

18 Zhao I, Bogossian F, Turner C. The effects of shift work and interaction between shift work and overweight/obesity on low back pain in nurses: results from a longitudinal study. J Occup Environ Med 2012;54:820-5

19 Kc R, Li X, Forsyth CB, et al. Osteoarthritis-like pathologic changes in the knee joint induced by environmental disruption of circadian rhythms is potentiated by a high-fat diet. Sci Rep 2015;5:16896

20 Numaguchi S, Esumi M, Sakamoto M, et al. Passive cigarette smoking changes the circadian rhythm of clock genes in rat intervertebral discs. J Orthop Res 2016:34:39-47.

21 Yoo SH, Yamazaki S, Lowrey PL, et al. PERIOD2::LUCIFERASE real-time reporting of circadian dynamics reveals persistent circadian oscillations in mouse peripheral tissues. Proc Natl Acad Sci USA 2004;101:5339-46.

22 Freemont AJ, Peacock TE, Goupille P, et al. Nerve ingrowth into diseased intervertebral disc in chronic back pain. Lancet 1997;350:178-81.

23 Brown SA, Pagani L, Cajochen C, et al. Systemic and cellular reflections on ageing and the circadian oscillator: a mini-review. Gerontology 2011;57:427-34.

24 Davidson AJ, Yamazaki S, Arble DM, et al. Resetting of central and peripheral circadian oscillators in aged rats. Neurobiol Aging 2008;29:471-7.

25 Gossan N, Zeef L, Hensman J, et al. The circadian clock in murine chondrocytes regulates genes controlling key aspects of cartilage homeostasis. Arthritis Rheum 2013:65:2334-45.

26 Yeung CY, Gossan N, Lu Y, et al. Gremlin-2 is a BMP antagonist that is regulated by the circadian clock. Sci Rep 2014;4:5183.

27 Molinos M, Almeida CR, Caldeira J, et al. Inflammation in intervertebral disc degeneration and regeneration. J $R$ Soc Interface 2015;12:20150429.

28 Guo B, Yang N, Borysiewicz E, et al. Catabolic cytokines disrupt the circadian clock and the expression of clock-controlled genes in cartilage via an NFKB-dependent pathway. Osteoarthritis Cartilage 2015;23:1981-8.

29 Hughes ME, Hogenesch JB, Kornacker K. JTK_CYCLE: an efficient nonparametric algorithm for detecting rhythmic components in genome-scale data sets. J Biol Rhythms 2010;25:372-80

30 McMahon JA, Takada S, Zimmerman LB, et al. Noggin-mediated antagonism of BMP signaling is required for growth and patterning of the neural tube and somite. Genes Dev 1998;12:1438-52.

31 Vo NV, Hartman RA, Yurube T, et al. Expression and regulation of metalloproteinases and their inhibitors in intervertebral disc aging and degeneration. Spine J 2013:13:331-41.

32 Bunger MK, Walisser JA, Sullivan R, et al. Progressive arthropathy in mice with a targeted disruption of the Mop3/Bmal-1 locus. Genesis 2005;41:122-32.

33 Dudek M, Gossan N, Yang N, et al. The chondrocyte clock gene Bmal1 controls cartilage homeostasis and integrity. J Clin Invest 2016;126:365-76.

34 Luoma K, Riihimäki H, Luukkonen R, et al. Low back pain in relation to lumbar disc degeneration. Spine 2000;25:487-92.

35 Agrawal A, Guttapalli A, Narayan S, et al. Normoxic stabilization of HIF-1alpha drives glycolytic metabolism and regulates aggrecan gene expression in nucleus pulposus cells of the rat intervertebral disk. Am J Physiol Cell Physiol 2007:293:C621-31. 


\section{Basic and translational research}

36 Rannou F, Lee TS, Zhou RH, et al. Intervertebral disc degeneration: the role of the mitochondrial pathway in annulus fibrosus cell apoptosis induced by overload. Am J Pathol 2004;164:915-24.

37 Lee HW, Kim SY, Kim AY, et al. Adiponectin stimulates osteoblast differentiation through induction of COX2 in mesenchymal progenitor cells. Stem Cells 2009;27:2254-62.

38 Wang $\mathrm{H}$, Liu H, Zheng ZM, et al. Role of death receptor, mitochondrial and endoplasmic reticulum pathways in different stages of degenerative human lumbar disc. Apoptosis 2011;16:990-1003.

39 Spengler ML, Kuropatwinski KK, Comas M, et al. Core circadian protein CLOCK is a positive regulator of NF-кB-mediated transcription. Proc Natl Acad Sci USA 2012;109:E2457-65.

40 Haas S, Straub RH. Disruption of rhythms of molecular clocks in primary synovial fibroblasts of patients with osteoarthritis and rheumatoid arthritis, role of IL-1 $\beta / T N F$ Arthritis Res Ther 2012;14:R122.

41 Shi J, Schmitt-Talbot E, DiMattia DA, et al. The differential effects of IL-1 and TNF-alpha on proinflammatory cytokine and matrix metalloproteinase expression in human chondrosarcoma cells. Inflamm Res 2004;53:377-89.
42 Tetlow LC, Adlam DJ, Woolley DE. Matrix metalloproteinase and proinflammatory cytokine production by chondrocytes of human osteoarthritic cartilage: associations with degenerative changes. Arthritis Rheum 2001;44:585-94.

43 Hoyland JA, Le Maitre C, Freemont AJ. Investigation of the role of IL-1 and TNF in matrix degradation in the intervertebral disc. Rheumatology (Oxford) 2008;47:809-14.

44 Le Maitre CL, Hoyland JA, Freemont AJ. Catabolic cytokine expression in degenerate and herniated human intervertebral discs: IL-1beta and TNFalpha expression profile. Arthritis Res Ther 2007;9:R77.

45 Storch KF, Paz C, Signorovitch J, et al. Intrinsic circadian clock of the mammalian retina: importance for retinal processing of visual information. Cell 2007; 130:730-41.

46 Sakai K, Hiripi L, Glumoff V, et al. Stage-and tissue-specific expression of a Col2a1-Cre fusion gene in transgenic mice. Matrix Biol 2001;19:761-7.

47 Sládek $M$, Rybová $M$, Jindráková $Z$, et al. Insight into the circadian clock within rat colonic epithelial cells. Gastroenterology 2007;133:1240-9.

48 Sive Jl, Baird $\mathrm{P}$, Jeziorsk $\mathrm{M}$, et al. Expression of chondrocyte markers by cells of normal and degenerate intervertebral discs. Mol Pathol 2002;55:91-7. 